\title{
Gamification in Online Educational Systems
}

\author{
Hazem Ibrahim ${ }^{1}$, Walid Ibrahim ${ }^{2}$ \\ ${ }^{1}$ Department of Compuer Sceince, University of Toronto, Canada, ${ }^{2}$ Department Computer \\ and Network Engineering, UAE University, UAE.
}

\begin{abstract}
The usage of gamification in online educational systems hasgrown considerably over the last several decades. This has been in response to reports of low completion and retention rates in online educational systems such as MOOCs. The usage of gamification and the application of social and educational networking to online education systems has been found to increase student satisfaction with the system. However, it has been shown that as students become more familiar with the gamified educational system, the initial boost in motivation diminishes. Efforts made in order to increase the personalization of gamified educational system, however, indicated an increase in student motivation over the duration of the course. Suggestions for further work include the usage of smarter leaderboard systems, as well as the potential for personalization in peer to peer online tutoring platforms.
\end{abstract}

Keywords: e-learning, gamificationas, MOOCs . 


\section{Gamification}

Before diving into the applications of gamification in the world of education, it would be wise to first understand what gamification entails. Deterding et al (2011) coined the term gamification as the use of game design elements in non-game contexts. They argue that gamefulness can be seen as a distinct but complementary term to playfulness. Looking at Caillois (2001) concepts of paidia (or "playing") and ludus (or "gaming") as poles of play activities, there is a clear distinction between the act of playing and gaming. Games are typically characterized by clear rules that govern the possible interactions and capabilities the user has within the game, as well as clear objectives which the user aims to accomplish. In addition, games often involve some sort of limitation, manifested in many different forms such as time or resource constraints, which the user must overcome.

Playing, on the other hand, lends itself more to free-form, improvisational and expressive behaviors and meanings. Playing, in other words, is a much broader category, which encompasses gaming. The fundamental characteristics of games include selfrepresentation with avatars, three-dimensional environments, and narrative context among others (Reeves et. al 2009). However, any of these characteristics can be found outside of the context of games and would not be characterized as 'gameful'. Therefore, a game can be defined as an amalgamation of these characteristics, through the lens of an environment bounded by rules and limitations. Gamified applications are clearly distinct from full-fledged games however, as these applications tend to incorporate some characteristics of games but cannot be classified as complete games. Moreover, this distinction can be subjective, as the definition of a 'game' tends to go beyond the properties of the game itself and includes the socially constructed meanings situated in the game. The definition of gamification can be restricted to the application of the fundamental elements thatare characteristic of games, or those that are readily associated with games and play an important role in gameplay, such as badges, leaderboards, and achievements, in a non-game context. In addition, another distinction between gamified applications and games is that the state in which the gamified application is used lives on the boundary between a game context and non-game context. In other words, gamified systems often transition between gameful experience, and other modes of engagement with the system. Games are only experienced in a gaming context, while gamified applications can be experienced in both a game and non-game context simultaneously.

Hence, the purpose of gamification is to improve user experience and user engagement in non-game services and applications, through the utilization of fundamental game design elements. Gamified applications have been used in a variety of different fields including education, finance, health, and sustainability, and has also been a key point in research for the development of engaging workplaces as well as mass-collaboration projects. 


\section{Online Education}

Over the last decade, the number of students utilizing online education platforms has been steadily increasing. Bates (2018) defines the term online course as "A form of distance education where the primary delivery mechanism is via the internet. These could be delivered synchronously or asynchronously. All instruction is conducted at a distance.", where distance education refers to classes that are not held in a classroom or on campus. Canada is considered to be a specifically mature market, as most institutions have been offering online courses for over 15 years (Bates 2018). While conducting a survey to determine the number of students taking some form of an online course in Canada in 2017, Bates concluded that approximately 16 percent of all course enrollments were for online courses. In addition, the number of universities offering online courses has increased by 11 percent from 2011 to 2016. The delivery mechanism of online education by universities has been diverse in nature. Approximately 25 percent of institutions utilized technologies for synchronous delivery, in the form of interactive lectures or webinars. However, more advanced technologies such as adaptive learning, artificial intelligence, and competencybased learning have yet to been implemented in Canadian institutions.

Surprisingly, Massive Online Open Courses (MOOCs) have not been widely implemented in online education by universities in Canada, with under 20 percent of institutions offering a MOOC in 2017. MOOCs have shown a rapid increase in growth and utilization since 2008. MOOCs typically involve short video lectures, computer-graded tests, and online discussion forums. Over 35 million students have enrolled in a MOOC in 2015, according to a study conducted by Kim (2016). Coursera, which is the most well-known MOOC platform, accounts for almost half of the students enrolled in MOOCs. The guiding principles by which MOOCs operate push forward the idea of democratized education, in which everyone has access to free education with unlimited participation and open access. Students also now have the option to earn degrees and certificates through MOOCs, as many major MOOC providers offer course credits through paid credentials, moving away from the free model established earlier. However, one of the main issues that plague MOOCs is the lack of personalization afforded to students on the platform. This depersonalization of education has given way to low student motivation and retention, generating low course completion totals in MOOCs.

The remainder of this paper will discuss current efforts by researchers to gamify online education platforms, as well as efforts made to personalize gamified educational systems. An analysis of the advantages and disadvantages of such systems will be conducted in the following section, followed by suggestions for future work in the field. 


\section{State-of-the-Art}

As mentioned previously, there is a significant difference between instructional games, and gamified educational systems. Creating instructional or educational games is a difficult, costly, and time-consuming process, which is limited in effectiveness as only a single set of learning objectives can be targeted by the game designer. In gamified educational systems however, game thinking and game design elements are utilized to improve learners' motivation and engagement with a regular online learning system. Game design elements, such as badges and ranks have been used prior to their usage in games, particularly in the military, where these badges were often used in place of monetary incentives. These game design elements have been shown to have the capacity to shape user behavior in a desirable direction, as shown by Dicheva et al. (2015).

According to Dominguez et al. (2013), since games motivate players by impacting their cognitive, emotional, and social areas, gamified educational systems should target these areas as well. In regard to cognition, games provide a series of cycles of expertise, in which the user is faced with a series of short-term objectives that they must complete in order to gain the necessary skill level required. In addition, the ordering of different tasks is nonlinear, giving the user freedom to decide their path towards accomplishing the objective. Similarly, these cycles of expertise can be utilized in course design.

Games tend to impact the emotion of their players by encouraging them to complete tasks which involve overcoming difficulty, where players often fail. Hence, reward systems are implemented to give players immediate recognition for their efforts, in the form of awards or points. If the difficulty of the tasks a user faces is balanced, this leads to high motivation towards the completion of the objectives. Finally, socially, games offer a multitude of avenues for players to interact with one another to cooperate and accomplish different objectives. These interactions allow players to build in-game identities which contribute significantly towards participation in the game. The effect of games on these three areas can be translated into gamified online educational platforms to increase student motivation and participation.

Dominguez et al. attempted to incorporate these areas into the design of a gamified educative experience for the "Qualification for users of ICT" course. In order to stimulate the cognitive area of students, a hierarchy of course topics and optional exercises was established. The first layer of the tree included the student's list of topics, while the second layer included optional exercises for each topic. The third layer matches specific tasks for each exercise, while the fourth and final layer includes specific steps to complete each task. As students complete their path through this tree, different trophies are awarded to the students based on the difficulty of the task. Direct evaluation of the completion of a certain task was done through students uploading screenshots of their work, as the tasks completed 

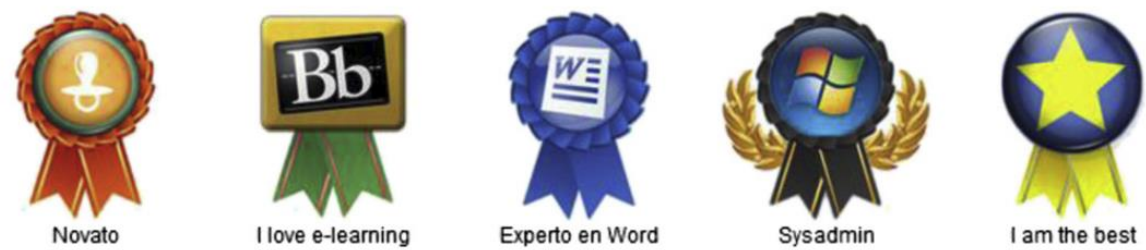

Figure 1. Special achievements represented as medals

were done using an external software, rather than non-immediate feedback where teachers evaluate student progress.

To incorporate the stimulation of the emotional area of students into the design of this course, Dominguez et al. utilized achievements as the form of reward system used in the course. In addition to regular achievements that are earned through completing the tasks defined by the system, special achievements in the form of medals were also incorporated to represent excellence in a certain task. Hidden achievements are also awarded by surprise when special tasks are completed. An example of the achievements awarded in the system can be seen in Figure 1. Finally, in regard to the social area of the system, Dominguez et al. created a leaderboard system to be used in the course, which ranks students based on the number of achievements they have completed. This leaderboard allows students to compete towards higher rankings by completing more tasks and exercises, which allows students to challenge themselves to obtain the most difficult and exclusive achievements.

Results from this experiment indicated that students who were part of the experimental group had significantly better results on all tasks that involved a practical application of the concepts taught in the course. However, these students also had lower scores on written examinations and participation than students in the control group. This drop in participation when using the gamified educational experience can be attributed to the idea of alienation through technology. Activities that build individual competence acquisition also cause students to separate from reality, thus lowering their interactions with other students. This, according to Heidegger (1977) only occurs when technology enframes the student or treats them as an object rather than a subject.

\section{Discussion}

While the usage of game design elements in educational settings have generally proven to improve student engagement and motivation, some researchers suggest that this increase in motivation is extrinsic rather than intrinsic in nature. Intrinsic motivation occurs when a student's desire to learn comes from within the student, rather than occurring as a result of an outside force. Offering badges and trophies as a result of completing a task may shift the 
motivations of a student from intrinsic to extrinsic. The result of such a shift is that once the reward is removed or completed, there is no longer a reason to perform the same behaviour again. This specifically occurs in the case that one completes a task that they would already freely choose to do as rewards are seen to be controlling and a hindrance of intrinsic motivation.

In addition, due to the utilization of leaderboards in gamified educational systems, social comparison is a regular occurrence. However, when individuals compare themselves with those who are worse or better than themselves, this can lead to feelings of superiority or low self-esteem respectively. However, leaderboards can also foster competition between individuals, which can lead to an increase in motivation. The negative effects of leaderboards and competition depend on the context by which the competition is viewed. Constructive competition occurs when the challenge is fun and allows for the individual to grow positive relationships with others, while destructive competition can lead to a decrease in intrinsic motivation. However, since leaderboards naturally highlight those at the top, they often lead to destructive competition and negative outcomes (Fülöp, 2009).

In an effort to study the negative effects of gamification, Hanus and Fox (2011) conducted a longitudinal study to determine the effect of gamification elements such as leaderboards, badges, and incentive systems on student satisfaction, motivation, enjoyment and grades over time. The results from this study suggested that these game design elements may be harmful to some educational outcomes. Students who participated in the gamified class were found to be less intrinsically motivated, which caused lower final exam scores. Since the class used in this experiment was an elective class, which students voluntarily enrol in at their own interest, the addition of these game design elements was found to be controlling and forced. Hence, students in the gamified class were less confident, and less satisfied with the course overall.

It should be noted that the gradual decrease in motivation that has been highlighted in the mentioned studies can likely be attributed to the novelty of gamified educational systems. Since this type of system is new to many of the students who participated in these studies, motivation and engagement is likely to drop as students become more familiar with the system.

\section{Future Work}

One of the newer types of online educational systems is the peer to peer online tutoring systems, which connects students with teachers on a one-to-one basis. Such systems include the "Tutorful" system, based in the UK. The system allows students and teachers to either complete a tutoring session in person, or online via video conferencing. Since this system aims to connect teachers to students directly, there is great potential for personalization, 
since the social and educational dynamic of one-to-one learning greatly differs from the dynamics of classroom-based teaching environment or a many-to-one environment exhibited in many online educational platforms. One possible way for personalizing such a system is a matching system which connects students and teachers together. Learning styles can be classified into more categories including: Activists, Reflectors, Theorists, and Pragmatists (Pritchard, 2013). Since different students optimally learn in different ways, if students and teachers were to take a cognitive assessment test, in order to determine their cognitive styles, the learning and teaching style of the student and teacher respectively can be matched optimally in order to enable the best possible learning environment for the student.

Such a system, given its relative novelty, could invoke many research questions, such as studying the effect of different matching algorithms on the performance and satisfaction of students, or an assessment of the different teaching styles that can be used to cater for individual students. In addition, the optimal number of students to teacher in a synchronous educational system could be studied, in order to determine whether the benefits of one-toone education, such as real- time question answering and personalization, can be extended to one-to-many effectively. Such a system would also be able to cater to students with learning disabilities, such as Dyslexia or Autism, automatically matching them with teachers who have the necessary qualifications to teach them.

One of the possible research questions that should be analyzed is the effect of different types of leaderboards in a gamified educational system. Currently, research has been done in order to embed game design elements into educational systems to increase student motivation through competition, however, this implementation can be improved by shaping the context in which the leaderboard exists in the system. The competition that is bred from the usage of a leaderboard is two-fold, depending on the perception of the user to the leaderboard. If the user views competition through the lens of "wanting to win", this affects their levels of motivation positively. However, if the user views the competition as "wanting to avoid losing", this has been found to have a negative effect on goal completion.

\section{Conclusion}

The application of game design elements into online educational systems has been shown to have temporary positive effects on student motivation and participation rates. Similarly, the implementation of social networking elements in gamified applications have also been found to encourage student motivation and cooperation within the online course. Several frameworks have emerged that specify the design process of gamified educational systems, with an emphasis on task design, which has been used in many gamified online applications. 
However, as the novelty of the gamified educational system diminishes, the participation rates of students tends to regress towards the mean. Many efforts have been made in order to improve student retention, including the personalization of the gamified course experiences through classifying students into different groups by their personality types, which has been shown to increase student retention rates.

Future work in this field of research includes the capacity for more intelligent personalization mechanisms, particularly in more novel educational systems such as peer to peer tutoring platforms. In addition, more research can be conducted to study the effect of different implementations of leaderboards on student motivation rates, including the implementation of seasonal leaderboards.

\section{References}

Bates, Tony (2018). The 2017 national survey of online learning in Canadian postsecondary education: methodology and results. International Journal of Educational Technology in Higher Education 15.1 (2018): 29.

Caillois, Roger (2001). Man, play, and games. University of Illinois Press, 2001.

Deterding, Sebastian, et al. (2011). From game design elements to gamefulness: defining gamification. Proc. 15th International Academic MindTrek conference: Envisioning future media environments. ACM, 2011.

Dicheva, Darina, et al (2015). Gamification in education: A systematic mapping study. Journal of Educational Technology \& Society 18.3.

Domínguez, Adrián, et al. (2013). Gamifying learning experiences: Practical implications and outcomes. Computers \& Education 63: 380-392.

Fülöp, Márta. (2009). Happy and unhappy competitors: What makes the difference? Psihologijske teme 18.2 (2009): 345-367.

Hanus, Michael D., and Jesse Fox (2015). Assessing the effects of gamification in the classroom: A longitudinal study on intrinsic motivation, social comparison, satisfaction, effort, and academic performance. Computers \& Education 80 (2015): 152- 161.

Heidegger, Martin (1977). The question concerning technology, and other essays. Garland Publishing Corp.

Kim, Sung-Wan (2016). "MOOCs in higher education." Virtual Learning. InTech, 2016.

Pritchard, Alan (2014). Ways of learning: Learning theories and learning styles in the classroom. Routledge, 3rd edition, ISBN-10: 041583493.

Reeves, Byron, and J. Leighton Read (2009). Total engagement: How games and virtual worlds are changing the way people work and businesses compete. Harvard Business Press. 